\title{
Observation of giant photocurrent gain in highly doped (In, Ga)N/GaN multiple-quantum-well-based photodiodes
}

\author{
C. Rivera ${ }^{a)}$ and E. Muñoz \\ ISOM and Dpto. Ingeniería Electrónica, ETSI Telecomunicación, Universidad Politécnica de Madrid, \\ Ciudad Universitaria, 28040 Madrid, Spain
}

(Received 11 March 2008; accepted 17 May 2008; published online 13 June 2008)

\begin{abstract}
The authors report on the observation of a giant photocurrent gain $\left(>10^{6}\right)$ in highly doped (In, Ga)N/GaN multiple-quantum-well-based photodiodes, which decreases with temperature. This large photocurrent gain appears at low forward bias and strongly depends on the current transport mechanism. Results of capacitance-voltage measurements show that the photocurrent gain is related to the screening of the built-in electric field by charge accumulation. The role of the $(\mathrm{Al}, \mathrm{Ga}) \mathrm{N}$ electron blocking layer in current transport has been studied by comparing samples nominally identical except for the existence of this layer. (C) 2008 American Institute of Physics.
\end{abstract}

[DOI: 10.1063/1.2942384]

Quantum structures have been proposed to improve the performance of devices in a large variety of applications including light emission (e.g., light emitting diodes or lasers), photodetection [e.g., quantum-well infrared photodetectors (QWIPs) or electrooptical modulators] and high-frequency electronics (e.g., high electron mobility transistors or resonant tunneling transistors). ${ }^{1-5}$ In the field of photodetectors, the advantages provided by quantum structures have been mainly used to control the spectral response of this type of devices. However, although other advantages such as the enhancement in the noise factor for avalanche photodiodes by introducing superlattice structures have been considered, 6,7 there are very few reports studying the possibility to exploit quantum structures in terms of responsivity. 8,9 Namely, Capasso et al. identified a mechanism, known as effective mass filtering, which leads to large photocurrent gain and, in turn, very high responsivity. ${ }^{8}$ This gain mechanism resembles that of a standard photoconductive gain mechanism, but, in this case, the gain is controlled by the ratio between the transit time for electrons and holes in the superlattice. Other subtle mechanism of photocurrent gain in piezoelectric materials enhanced by the use of quantum-well (QW) structures was recently pointed out by Rivera et al., based on the photoinduced screening of the negative average electric field formed by the built-in electric fields. ${ }^{10}$ It is also worth noting that large photocurrent gains have been observed in quantum dot and quantum wire-based photodetectors. ${ }^{11,12}$ In this work, we report experimental results on GaN-based $p$ - $n$ junction photodiodes with an (In, Ga)N/GaN multiple-quantum-well (MQW) structure embedded in the $n$-type side of the junction. These results indicate that a huge gain can be achieved using these structures while dark current can be kept low. The characteristics and origin of the photocurrent gain at reverse and forward biases are discussed.

Two samples grown on $c$-face sapphire substrates by metal-organic vapor-phase epitaxy were studied. Wafers were grown by TopGaN. In both samples, the MQW region consists of a five-period structure with 3-nm-thick wells and 14-nm-thick barriers. The In content in the QWs determined from photoluminescence and photocurrent measurements is

\footnotetext{
${ }^{a)}$ Electronic mail: crivera@die.upm.es.
}

about $8 \%$. Carrier concentrations in the $p$-type and $n$-type layers are $6 \times 10^{17}$ and $4 \times 10^{18} \mathrm{~cm}^{-3}$ respectively. The $\sim 0.3$ - $\mu$ m-thick $p$-type layer was doped with $\mathrm{Mg}$, whereas the $\sim 2.8$ - $\mu$ m-thick $n$-type layer was doped with Si. Barriers in the MQW region were also doped with Si providing an electron concentration as high as $3 \times 10^{18} \mathrm{~cm}^{-3}$. In one of the samples (sample I), a 20-nm-thick $\mathrm{Al}_{0.12} \mathrm{Ga}_{0.88} \mathrm{~N}$ barrier was deposited on the $p$-type side on top of the MQW region. The other sample (sample II) is nominally identical except for the AlGaN electron blocking barrier. Photodetectors fabricated from both samples have an active region of $300 \times 300 \mu \mathrm{m}^{2}$. A semitransparent $(\sim 65 \%$ transparency) $\mathrm{Ni}(5 \mathrm{~nm}) / \mathrm{Au}$ $(5 \mathrm{~nm})$ contact was deposited to allow front illumination, whereas Ohmic contacts to the $p$-type and $n$-type layers were made of $\mathrm{Ni}(5 \mathrm{~nm}) / \mathrm{Au}(200 \mathrm{~nm})$ and $\mathrm{Ti}(5 \mathrm{~nm}) / \mathrm{Al}$ $(200 \mathrm{~nm})$, respectively. Current-voltage $I-V$ characteristics in dark conditions and under illumination were measured using an Agilent 4156C parameter analyzer. Capacitance and conductance measurements were performed using a standard HP4284 LCR meter with a $50 \mathrm{mV}$ test signal at different frequencies. Optical excitation was carried out using the light of a $150 \mathrm{~W}$ Xe arc lamp filtered by a monochromator.

Devices fabricated from both samples exhibit responsivities higher than $e / h v$ (i.e., photocurrent gain), where $e$ is the electronic charge and $h v$ is the photon energy, at room temperature and forward bias under optical excitation with energies above the QW band gap (cf. Fig. 1). The photocurrent

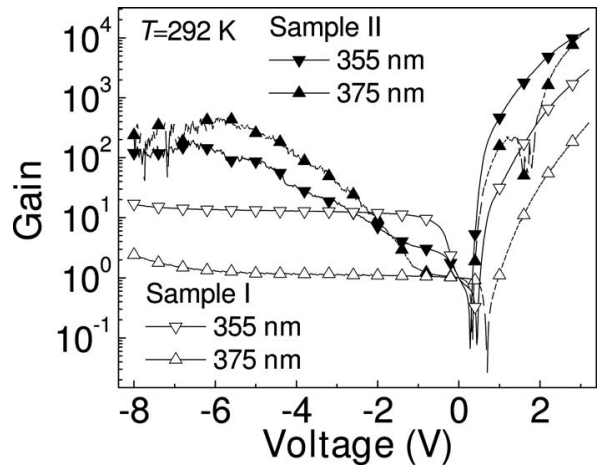

FIG. 1. Normalized photocurrent gain for both samples I and II under optical excitation in the wells $(375 \mathrm{~nm})$ and barriers $(355 \mathrm{~nm})$. 


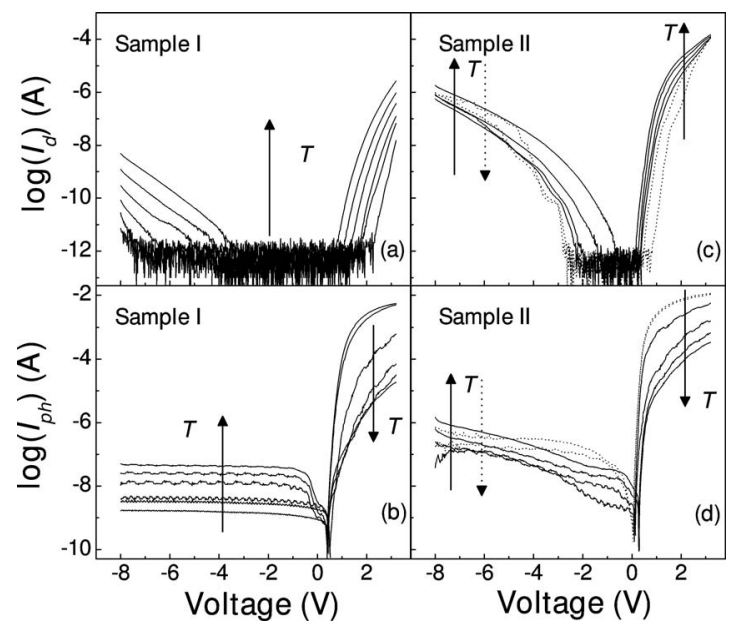

FIG. 2. $I-V$ characteristics as a function of temperature $T$ for sample I (sample II) (a) [(c)] in dark $\left(I_{d}\right)$ and (b) $[(d)]$ under optical excitation $\left(I_{\mathrm{ph}}\right)$ at $\lambda_{\mathrm{ex}}=355 \mathrm{~nm}$. Temperature ranges from 250 down to $10 \mathrm{~K}$ in steps of $50 \mathrm{~K}$. The arrow shows the direction of current growth as $T$ increases for forward and reverse biases. The dashed arrows show this variation below $100 \mathrm{~K}$ at reverse bias.

gain in this figure is given by $G(V)=I_{\mathrm{ph}}(V) / I_{\mathrm{ph}}(V=0)$, whereas $I_{\mathrm{ph}}$ is the photocurrent and $V$ is the applied voltage.

The results of Fig. 1 show four striking features. Firstly, the photocurrent gain is strongly sensitive to applied voltage, especially at forward bias. Secondly, the photocurrent gain in sample II is larger than in sample I, probably due to the effect of the AlGaN blocking barrier, as it will be discussed below. This reduction in the photocurrent gain is more important at reverse bias, where the photocurrent gain has a flat response. Thirdly, the photocurrent gain shows a large dependence on the excitation wavelength either in the barriers $\left(\lambda_{\text {ex }}=355 \mathrm{~nm}\right)$ or in the QWs $\left(\lambda_{\mathrm{ex}}=375 \mathrm{~nm}\right)$ in the case of sample I (the emission efficiency of the Xe lamp is approximately the same at these two wavelengths). In sample I, the AlGaN blocking barrier hinders the minority carrier transport in the $p$-type region. Finally, the sign of photocurrent is reversed above a certain forward voltage. This behavior is unusual for $p-n$ junction photodiodes based on bulk material. Similar results were nevertheless found in $p-n$ junction photodiodes with a superlattice structure in the space charge region (SCR) of the device. ${ }^{8,13}$ Note that the structure studied in Ref. 8 is slightly different to the one studied here, since barriers in the superlattice have a larger band gap than the $p$ and $n$-layers. Moreover, in contrast to our results, the change in the sign of photocurrent is reached at the built-in voltage. Other reports on GaAs/AlAs $n^{+}-p^{-}-n^{+}$structures also showed that giant photocurrent gain can be achieved using superlattices as active detector regions, but in this case the origin of the gain was attributed to recombination and trapping processes. ${ }^{14}$

A detailed study of the $I-V$ characteristics as a function of temperature allows us to determine two different photocurrent gain regimes for forward and reverse biases, respectively (cf. Fig. 2). The photocurrent increases monotonously as temperature decreases at forward bias in both samples, reaching large values of photocurrent gain. Namely, the photocurrent gain $G(V=1.5 \mathrm{~V})$ measured at $10 \mathrm{~K}$ is as high as $1.1 \times 10^{6}\left(7.4 \times 10^{6}\right)$ in sample I (sample II), which, in turn, corresponds to a responsivity of about $1000 \mathrm{~A} / \mathrm{W}$ $(5000 \mathrm{~A} / \mathrm{W})$. At the same time, dark current decreases, reAuthor complimentary copy. Redistribution subject to AIP

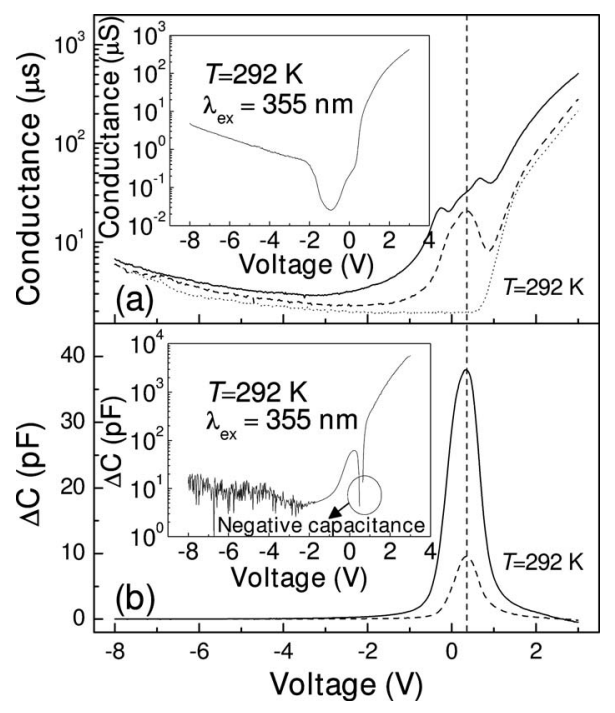

FIG. 3. Photoconductance and photocapacitance as a function of voltage for optical excitation in the wells $\left(\lambda_{\mathrm{ex}}=375 \mathrm{~nm}\right)$ and barriers $\left(\lambda_{\mathrm{ex}}=355 \mathrm{~nm}\right)$ at room temperature and $f_{\text {test }}=1 \mathrm{MHz}$. The inset shows the photoconductance and photocapacitance at $f_{\text {test }}=1 \mathrm{kHz}$.

sulting in a photocurrent/dark current contrast ratio in excess of $10^{5}$ (maximum value in excess of $10^{8}$ at $\sim 1 \mathrm{~V}$ ) under optical excitation for $\sim 1 \mathrm{~mW} / \mathrm{cm}^{2}$. Similar results were obtained for selective optical excitation in the QWs.

The photocurrent at reverse bias follows a different temperature dependence. On the one hand, the photocurrent exhibits the same behavior as the dark current in both samples. On the other hand, the photocurrent for sample I decreases as a function of temperature in the whole temperature range measured, whereas photocurrent for sample II decreases from room temperature to $75 \mathrm{~K}$ and, subsequently, increases from 75 to $10 \mathrm{~K}$. The later increase seems to be related to an improvement in the tunneling current in the MQW region [see the steps in dark current below $75 \mathrm{~K}$ in Fig. 2(a)]. The photocurrent gain at reverse bias is limited to values between 100 and 1000 at room temperature for sample II.

To understand the photocurrent gain mechanism, we have simultaneously measured photodetector capacitance and conductance in dark and under optical excitation. Results for sample II are shown in Fig. 3 (similar results were obtained for sample I). For a test frequency of $1 \mathrm{MHz}$, both photocapacitance characteristics and the conductance under optical excitation exhibit a pronounced resonance at $0.325 \mathrm{~V}$. A change in the excitation wavelength affects the amplitude of this resonance and also its shape in the case of the conductance characteristics. Namely, the higher carrier generation for optical excitation above the barrier band gap $\left(\lambda_{\mathrm{ex}}=355 \mathrm{~nm}\right)$ accounts for the larger values of photocapacitance and conductance. We have also observed other features at lower test frequencies. In the inset of Fig. 3, photocapacitance characteristics show negative values for a test frequency of $1 \mathrm{kHz}$ in a short voltage range close to the resonance peak. The photocapacitance increases monotonously to reach large values for higher forward voltages. This large capacitance response indicates that charge is stored in steady state under optical excitation. In contrast to the results of photocapacitance, conductance tends to the same value regardless of the frequency at higher forward voltages.

If we compare results of Fig. 2 with those of Fig. 3, we can notice that the resonance peak in photocapacitance coinicense or copyright, see http://apl.aip.org/apl/copyright.jsp 


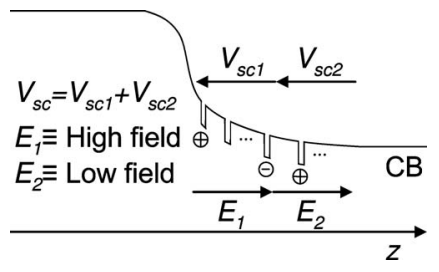

FIG. 4. Schematic representation of a generic conduction band (CB) for photoinduced screening. The electron (in the $\mathrm{CB}$ ) and hole (in the valence band) accumulation at the boundaries of the electric field domains is explicitly indicated.

cides with the minimum in photocurrent. Therefore, it seems that the change in the sign of photocurrent is related to the same mechanism responsible for the negative capacitance and the changes in conductance. These results lead to propose a model of photocurrent gain based on carrier-induced screening of the built-in electric field. Photogenerated carriers are accumulated at the opposite sides of the electric field domain formed in the MQW region inside the SCR decreasing the built-in electric field and, hence, increasing the forward current (see Fig. 4). The formation of electric field domains is possible since the QW region is highly doped $\left(\sim 5 \times 10^{12} \mathrm{~cm}^{-2}\right) .{ }^{15}$ The carrier-induced screening can be thus characterized by an effective voltage defined as

$$
V_{\mathrm{sc}} \approx n \frac{k T}{e} \ln \left(\frac{I_{\mathrm{ph}}}{I_{d}}\right)
$$

where $n$ is the ideality factor, which accounts for the transport mechanism, $k$ is the Boltzmann constant, $T$ is the temperature, and $I_{d}$ is the dark current. Equation (1) can be readily obtained following a similar procedure to that described in Ref. 16. In this context, Eq. (1) relates the photocurrent measured by the external circuit with the photoinduced screening, i.e., $V_{\mathrm{sc}}$ is the open circuit voltage associated with $I_{\mathrm{ph}}$. The large photocurrent gain at low temperatures is explained by Eq. (1). From Eq. (1), the ratio $I_{\mathrm{ph}} / I_{d}$ increases exponentially as temperature decreases for a fixed value of $V_{\mathrm{sc}}$. The exponential increase in photocurrent is limited by the series resistance or changes in the transport mechanism.

This model also allows the interpretation of photocapacitance results, including the negative capacitance. The negative capacitance phenomenon at low frequency has been reported in literature in a variety of structures including $p-i-n$ diodes and QWIPs. The physical mechanism behind the negative capacitance has been associated with different origins such as space charge effects, trapping processes, or contact injection, depending on the device properties. ${ }^{17}$ In this case, the initial drop in photocapacitance values is thought to be caused by a change in the stored charge and, therefore, in the $V_{\mathrm{sc}}$ dependence as a function of applied voltage. A phenomenological description of capacitance-voltage $(C-V)$ characteristics can be obtained from

$$
\frac{1}{C_{\text {light }}}=\frac{1}{C_{\text {dark }}}-\frac{\partial V_{\mathrm{sc}} / \partial W}{\partial Q_{p} / \partial W},
$$

where $C_{\text {light }}$ is the photocapacitance, $C_{\text {dark }}$ is the capacitance in dark conditions, $W$ is the SCR width, and $Q_{p}$ is the charge of the $p$-type region. In Eq. (2), we have assumed an asymmetric junction with a very high $p$-doping $\left(\sim 5 \times 10^{19} \mathrm{~cm}^{-3}\right.$ in our samples). In contrast to $\partial V_{\mathrm{sc}} / \partial W$, the sign of $\partial Q_{p} / \partial W$ remains constant with applied voltage. Hence, the changes in the slope of $C-V$ characteristics depend on the variation of $V_{\text {sc }}$ as a function of applied voltage, as mentioned before. The second term of Eq. (2) becomes more important at forward bias, as experimentally observed.

Other results already reported by Rivera et al. for nonintentionally doped (In, Ga)N/GaN MQW structures showed that collection efficiency of photogenerated carriers is affected by the dominant transport mechanism in the MQW region. ${ }^{18}$ In contrast to results presented here, neither $C-V$ features nor photocurrent gain were found at room temperature and low forward bias. Nevertheless, the behavior found at reverse bias is similar to that of sample I (both structures have an $\mathrm{AlGaN}$ electron blocking barrier). Therefore, we think that doping is responsible for the charge accumulation under optical excitation and, in turn, for the high photocurrent gain.

In summary, we have observed a giant photocurrent gain at low temperatures, which can be useful to develop photodetectors. From our results, there are two conditions to reach the observed photocurrent gain. The first one is that charge must be accumulated within the SCR. The second one is that transport mechanism must allow high current injection. The photocurrent gain is thus expected to be more efficient at forward bias and for highly doped MQW structures. The use of an $\mathrm{AlGaN}$ electron blocking barrier results in the partial inhibition of the photocurrent gain mechanisms in addition to decreasing the dark current both at forward and reverse biases.

The authors would like to thank the technical cooperation of P. Prystawko and M. Leszczynski. Partial financial support from project Futursen (CM-0505/AMB/0374) is acknowledged.

${ }^{1}$ Z. I. Alferov, Rev. Mod. Phys. 73, 767 (2001).

${ }^{2}$ R. E. Slusher, Rev. Mod. Phys. 71, S471 (1999).

${ }^{3}$ D. A. B. Miller, D. S. Chemla, T. C. Damen, C. Gossard, W. Wiegmann, T. H. Wood, and C. A. Burrus, Appl. Phys. Lett. 45, 13 (1984).

${ }^{4}$ T. Mimura, S. Hiyamizu, T. Fuji, and K. Nanbu, Jpn. J. Appl. Phys. 19, L225 (1980).

${ }^{5}$ F. Capasso, S. Sen, F. Beltram, L. M. Lunardi, A. S. Vengurlekar, P. R. Smith, N. J. Shah, R. J. Malik, and A. Y. Cho, IEEE Trans. Electron Devices 36, 2065 (1989).

${ }^{6}$ F. Capasso, W. T. Tsang, A. L. Hutchinson, and G. F. Williams, Appl. Phys. Lett. 40, 38 (1982).

${ }^{7}$ C. J. Summers and K. F. Brennan, Appl. Phys. Lett. 51, 276 (1987).

${ }^{8}$ F. Capasso, K. Mohammed, A. Y. Cho, R. Hull, and A. L. Hutchinson, Phys. Rev. Lett. 55, 1152 (1985).

${ }^{9}$ F. Capasso, G. Ripamonti, W. T. Tsang, A. L. Hutchinson, and S. N. G. Chu, J. Appl. Phys. 65, 388 (1988).

${ }^{10}$ C. Rivera, J. L. Pau, and E. Muñoz, Appl. Phys. Lett. 89, 263505 (2006).

${ }^{11}$ H. Lim, B. Movaghar, S. Tsao, M. Taguchi, W. Zhang, A. A. Quivy, and M. Razeghi, Phys. Rev. B 74, 205321 (2006).

${ }^{12}$ C.-Y. Lu, S.-J. Chang, S.-P. Chang, C.-T. Lee, C.-F. Kuo, H.-M. Chang, and Y.-Z. Chiou, Appl. Phys. Lett. 89, 153101 (2006).

${ }^{13}$ D. V. Lang, A. M. Sergent, M. B. Panish, and H. Temkin, Appl. Phys. Lett. 49, 812 (1986).

${ }^{14}$ A. Sibille, C. Minot, H. Le Person, J. F. Palmier, and F. Mollot, Europhys. Lett. 18, 619 (1992).

${ }^{15}$ H. T. Grahn, R. J. Haug, W. Müller, and K. Ploog, Phys. Rev. Lett. 67, 1618 (1991).

${ }^{16}$ S. M. Sze, Physics of Semiconductor Devices (Wiley, New York, 1981), pp. 291-292.

${ }^{17}$ M. Ershov, H. C. Liu, L. Li, M. Buchanan, Z. R. Wasilewski, and A. K. Jonscher, IEEE Trans. Electron Devices 45, 2196 (1998).

${ }^{18}$ C. Rivera, J. L. Pau, A. Navarro, and E. Muñoz, IEEE J. Quantum Electron. 42, 51 (2006). 